\title{
Performance Analysis of RTS/CTS Protocol over Basic Access in Wireless Networks
}

\author{
Study of Protocol Impact on Performance In Wireless Networks
}

\author{
Kuruvilla Mathew \\ Faculty of CS and IT \\ University Malaysia Sarawak (UNIMAS) \\ Kota Samarahan, Malaysia \\ kuruvila.mathew.k@ieee.org
}

\author{
Biju Issac \\ School of Computing \\ Teesside University \\ Middlesbrough, UK \\ bissac@ieee.org
}

\author{
Tan Chong Eng \\ Faculty of CS and IT \\ University Malaysia Sarawak (UNIMAS) \\ Kota Samarahan, Malaysia \\ cetan@ieee.org
}

\begin{abstract}
The CSMS/CA protocol is employed in wireless networks in order to overcome issues such as the hidden node problem. This mechanism is expected to handle collisions better using the RTS/CTS mechanism. This method will allow a participating node to take part in communication only if it receives a "Clear to Send" message and thereby, theoretically "avoiding" collision. The objective of this paper is to analyse the improvement that the RTS/CTS mode brings over the Basic Access mode. The paper presents the study of wireless nodes within a specific area with increasing node concentration to verify the performance impact of a protocol in wireless networks, particularly when the node concentration increases.
\end{abstract}

Keywords- CSMA/CA, 802.11b \& 802.11g, MAC Layer protocols, Pamvotis

\section{INTRODUCTION}

The digital society is growing, with millions of ubiquitous networking capable devices people are carrying around, most of the time unknowingly. As these devices are made to be extremely mobile and travel with the subjects that carry them, the only option to interconnect them is using wireless technology. On the other hand, we have rural geographic locations with difficult terrains[1], in which wired networks are difficult or expensive to install and maintain. The wireless network connectivity provides the best solution for such locations as well. However, wireless medium uses shared medium and therefore is prone to collision. The issue of collision increases drastically as the number of nodes increases as it increases the number of devices in the collision domain. This brings forward, the use of improved protocols like Carrier Sense Multiple Access (CSMA) with Collision Avoidance, specifically for wireless access of Media Access Control (MAC) layer. In this paper, we will study the effect of a good protocol in improvement of throughput for overall effectiveness of wireless communication. The objective of the study is to evaluate the impact a reasonably good protocol can have on wireless network performance.

This paper is organised into the following sections. Section II describes the related previous works. Section II describes the CSMA protocol in brief. Section IV presents the simulation tool used for the experiment. Section V describes the experiment and results and section VI presents the conclusion of the study.

\section{RELATED WORKS}

Mathew, K., Issac, B. presented an "ubiquitous text transfer using sound a zero-infrastructure alternative for simple text communication"[1], an idea to bring communication to difficult terrains. The research is expected progress towards design of optimal MAC layer protocol for this. Ye, W., Heidemann, J., \& Estrin, D. proposed "S-MAC, a medium-access control (MAC) protocol designed for wireless sensor networks, An energy-efficient MAC protocol for wireless sensor networks"[2]. S-MAC uses some novel techniques to reduce energy consumption and supports selfconfiguration.

Wang, G., et. al. analysed the delay performance of Basic Access and the RTS/RTC modes of the 802.11 protocol and presented their Delay analysis of the IEEE 802.11 DCF[3]. Bruno, R., Conti, M., \& Gregori, E. presented "IEEE 802.11 optimal performances: RTS/CTS mechanism vs. basic access"[4]. They analysed the throughput of the $802.11 \mathrm{~b}$ Wireless Local Area Networks (WLANs), deriving the throughput formula for the RTS/CTS Access method of the p-persistent IEEE 802.11b MAC protocol and validated the accuracy of the proposed model using simulated results. Bianchi, G. presented a performance analysis of the IEEE 802.11 distributed coordination function [5]. The paper presents a simple, accurate, analytical model to compute the 802.11 DCF throughput, with assumptions of finite number of terminals and ideal channel conditions. Nasipuri, A., Zhuang, J., \& Das, S. R. presented a multichannel CSMA MAC protocol for multihop wireless networks[6]. It describes a new carrier-sense multiple access (CSMA) protocol for multihop wireless networks, sometimes also called ad hoc networks, using a "soft" channel reservation, and show that they perform better than their single channel counterpart.

Dean, T., in his book Network+ guide to networks[7], describes the CSMA/CA among other Network topics. Xu, K., Gerla, M., \& Bae, S. discussed the Effectiveness of RTS/CTS handshake in IEEE 802.11 based ad hoc networks[8]. They suggest that the RTS/CTS mechanism is 
not effective in handling interferences created by hidden nodes outside the transmission range the receivers. Sobrinho, J. L., de Haan, R., \& Brazio, J. M. analyses why RTS-CTS is not the ideal wireless LAN multiple access protocol[9]. They discuss how, as it tries to solve the hidden-stations problem of CSMA, creates new problems derived from the interaction among its control and data packets and tries to systematically identify scenarios where the RTS/CTS mechanism deviates from the standard expected behaviours. Chatzimisios, P., Boucouvalas, A. C., \& Vitsas, V. analyses the Effectiveness of RTS/CTS handshake in reducing collision duration for 54 $\mathrm{Mbit} / \mathrm{s}$ by extending an existing mathematical model for the particular packet overheads and delays defined in 802.11a. and concludes, that the RTS/CTS scheme is not beneficial in most network scenarios for the $54 \mathrm{Mbit} / \mathrm{s}$ data rate[10].

Weinmiller, J., Woesner, H., Ebert, J. P., \& Wolisz, A. presented "Analyzing the RTS/CTS mechanism in the DFWMAC media access protocol for wireless LANs"[11]. The paper analyses the RTS/CTS mechanism, its problems, its effectiveness etc. $\mathrm{Yu}, \mathrm{C}$., et. al. discussed medium access control mechanisms in mobile ad hoc networks[12] on IEEE 802.11 mobile ad-hoc networks with multi-hop access and some issues they pose. They presented some techniques to enhance the channel utilisations. Ray, S., Carruthers, J. B., \& Starobinski, D. presented the RTS/CTS-induced congestion in ad hoc wireless LANs[13]. They propose a solution called RTS validation, for issues where the RTS/CTS mechanisms create congestions for packets when larger number nodes are involved. Their solution offers a $60 \%$ gain in the peak throughput.

Tay, Y. C., \& Chua, K. C. presented a study on capacity analysis for the IEEE 802.11 MAC protocol[14]. The paper uses an analytical model to study the channel capacity (maximum throughput) when using the basic access. They show that the probability of collision depends on the minimum Window Size and the number of nodes rather than packet size, latency, acknowledgement timeout, etc. Bianchi, G. presented IEEE 802.11-saturation throughput analysis[15]. He presented a simple analytical model to compute the saturation throughput performance in the presence of a finite number of terminals and in the assumption of ideal channel conditions. The model applies to both basic and RTS/CTS access mechanisms. Chatzimisios, P., Boucouvalas, A. C., \& Vitsas, V. discussed Packet delay analysis of IEEE 802.11 MAC protocol[16] by extending some throughput analysis in order to identify when the RTS/CTS mechanism achieves lower packet delay with respect to the Basic Access Mechanism. Kuo, W. K., Liu, F., \& Kuo, C. C. J. J. discussed an Enhanced backoff scheme in CSMA/CA for IEEE 802.11[17] by dynamically adjusting the contention window (CW) around the optimal value.

The Pamvotis simulation software[18] was used to conduct the simulation for this experiment. The software can be run out of the box for many standard scenarios and allows writing additional code to do specialised simulations. The results presented in this paper used the out of the box options for running the experiments.

\section{THE PROTOCOL - CSMA}

This paper studies the performance evaluation on the MAC Layer protocol for IEEE 802.11 Wireless LANs, the CSMA protocol with Basic Access and Collision Avoidance (CA) modes in particular. The basic access methods used in wired networks employ a Carrier Sense Multiple Access (CSMA) with Collision Detection (CD), which does not work well for wireless media as it poses unique challenges like the "hidden nodes" which are non-existent in wired mediums. Due to this, an adaptation of the protocol which applies CA over CSMA is employed in wireless (LAN) networks.

The CSMA/CA uses a method similar to token passing. A node wanting to utilise the channel sends a RTS packet to the central concentrator. The central concentrator responds with a CTS message when the channel is clear and the node starts transmission only after receiving this. This essentially leaves the control of media usage with the central concentrator and thereby eliminating any possibility of collision and hence called collision avoidance. This mechanism, of course, comes with an overhead, but is still expected to deliver overall better performance. As we see in fig. 1, even though the nodes, $\mathrm{A}, \mathrm{B}$ and $\mathrm{C}$ are within the range of the access point, they are outside the transmission range of each other. Hence a node wanting to transmit will not be able to "see" the other nodes in transmission and start transmitting, thereby causing collision. With the RTS/CTS mechanism in place, the node will first make an RTS request and start transmitting data only after a CTS message is received from the access point. Since the data communication is being co-ordinated by the central concentrator, theoretically, there are no more collisions and therefore "collision avoidance" is achieved.

This paper analyses the performance improvement of the RTS/CTS based protocol in comparison to the basic access mode as the concentration of nodes in a specific area increases. Since the experiment is only trying to verify that an effective protocol can achieve improvement, a simulation based approach will be suitable[7]. The simulation is carried out using the Pamvotis simulation software.

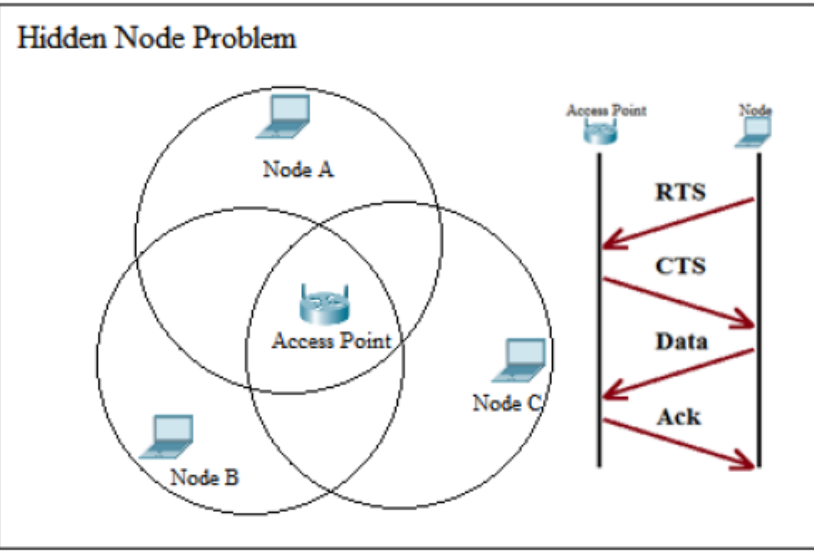

Fig. 1. The CSMA/CA and Hidden Node Problem 
The CSMA/CA is expected to tackle the "Hidden Node Problem" that is noticed in wireless networks. Wireless networks share the medium of access, namely the RF medium, presenting the problem of collision. Every additional node in the transmission range makes the collision domain larger and adds to the collision challenge. Even if the CSMA protocol is in use, wireless device in the extreme range of a central concentrator will not be able to sense another transmission happening on the central concentrator with a node on the other extreme range of the access point. This multiplies the number of collisions in a wireless paradigm, even with the use of the CSMA protocol. A simple "Collision Detection" (CD) and back of is not sufficient for efficient communication in this kind of scenarios. The wireless hidden node problem is better addressed using the CSMA/CA (carrier sense multiple access with collision avoidance) protocol. This employs a mechanism similar to token passing, where a node wanting to transmit will make a "Request To Send" (RTS) and wait till it receives a "Clear To Send" from the central concentrator. Therefore each node in the participating network is allowed to send data only once it receives a CTS message, thereby, theoretically eliminating the collision [7].

This experiment evaluates the improvement the protocol brings to wireless communication. The simulation for this experiment is carried out using the Pamvotis software[18].

\section{THE SIMULATION SOFTWARE}

The experiments for this study was conducted using the PAMVOTIS[18] simulation software. This was developed using Java, by Dr. Dimitris El. Vassis and Vassilis Zafeiris, a Telecommunication's Engineer and a Software Engineer respectively and supports a number basic features, including the hidden node terminal and supports the $802.11 \mathrm{~b}$ and g protocols out of the box. This supports multiple nodes which can be placed in an x,y coordinate plane and allows generation of a variety of traffic sources and some QoS parameters as well. This is open source and cross-platform, which increases its usability across scenarios.

The standard parameters we have selected for this study are listed as follows. We have selected to store 100 values per statistic and a simulation time of $300 \mathrm{sec}$, and standard QoS configuration. For individual node configuration, we used the option to upload an excel file to specify the following details. Node data rate, which is $54 \mathrm{kbps}$ for $802.11 \mathrm{~g}$ networks and $11 \mathrm{kbps}$ for $802.11 \mathrm{~b}$ nodes, packet length of 8000 bits for $802.11 \mathrm{~g}$ and 4000 bits for $802.11 \mathrm{~b}$ nodes, a mix of $20 \%$ exponential, $20 \%$ uniform and $60 \%$ constant packet length distribution. Each node is given a coverage range of $30 \mathrm{~m}$. The packet rate $(\mathrm{pkt} / \mathrm{sec})$ selected was 8 for $20 \%$ of the nodes, 5 for $20 \%$ of nodes and 4 for $60 \%$ of nodes. This pattern of node distribution was repeated for the number of nodes used for each experiment.

The software allows placing each of the nodes in an x,y co-ordinate plane. Nodes were placed in an equal space distribution in the plane covering a specific area, with a node concentration of $2,5,10,25,50$ and 100 nodes for each experiment. We are running the experiment for the Basic
Access mode and the RTS/CTS mode to evaluate the variation in performance the protocol brings to the scenario.

\section{THE EXPERIMENT AND RESULTS}

This section explores the various experiments and results that were conducted for this study. This is not a comprehensive study, but a study indicative of the improvements the RTS/CTS mechanisms widely employed in the CSMA/CA protocols. Here we look at the time to complete the simulation, various delays noticed like mean delay, jitter delay, etc. as indicators of improvements. Static parameters used for the simulation was using a mix of $802.11 \mathrm{~b}$ and $802.11 \mathrm{~g}$ with a $20 \%$ concentration of $802.11 \mathrm{~b}$ nodes for all experiments using 5 nodes or more. The experiment using 2 nodes used 1 each of $802.11 \mathrm{~b} \&$ g nodes. We recorded 100 values per statistic and took the mean values for our comparative study. The scenario used varying node concentrations in a specific area of consideration, on increasingly closer $\mathrm{x}, \mathrm{y}$ co-ordinates from 2 nodes to 100 nodes on a $100 \times 100$ meters plane.

The simulation software allows us to record a number of parameters, of which the following was chosen for our study, as they were suggestive enough for the intended analysis.

1. Simulation Completion Time: The actual time taken (in seconds) to actually complete the simulation was recorded for each of the experiment and compared.

2. Media access delay: The delay of a packet between the time it is picked up from the transmitter until it is successfully received to the receiver, including the retransmission delays and transmission delay.

3. Total packet delay: The total delay from the birth of a packet until its reception from the receiver, including queueing and media access delays.

4. Delay Jitter: The total delay jitter, the standard deviation of the total packet delay of each node.

5. Retransmission Attempts: The mean of retransmission attempts due to collisions until the packet is successfully transmitted.

\section{A. Simulation Completion Time (Run Time)}

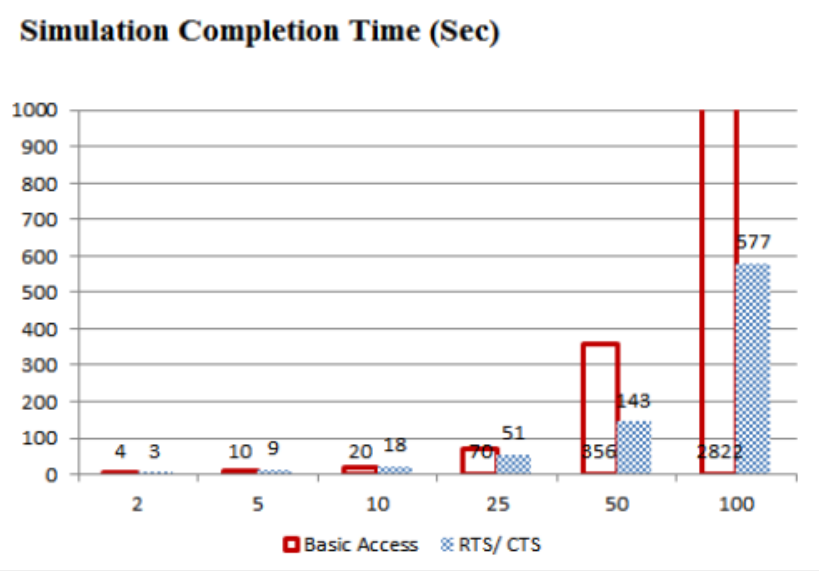

Fig. 2. Data Analysis: Simulation Completion Time in seconds 


\section{B. Media Access Delay}

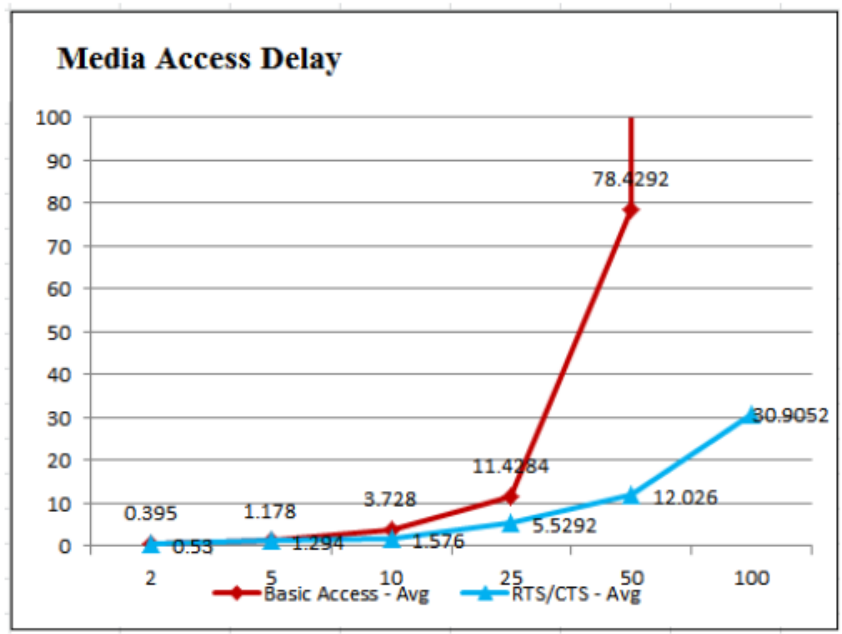

Fig. 3. Data Analysis: Media access delay in milliseconds

As we see in fig 2., the experiment with 2 and 5 nodes showed very little difference in the simulation times, but the experiment with 10 nodes was showing a clear advantage of using the RTS/CTS mechanism. The simulation too took considerably longer with 25,50 and 100 nodes. The experiment with 100 nodes using Basic Access mode was shooting way outside the chart plot area taking about 47 minutes to complete, probably due to large number of retransmissions resulting from too many collisions. The data shows that the RTC/CTS mode offers better performance.

The media access delay data for the experiment is shown in fig 3. We notice, quite interestingly, that for the first scenario using just 2 nodes, the media access delay is higher in the RTS/CTS mechanism, but as the node concentration increases, we see the delay increasing. The delay for the experiment with 100 nodes, as expected, has shot through beyond the range of the graph.

\section{Total Packet Delay}

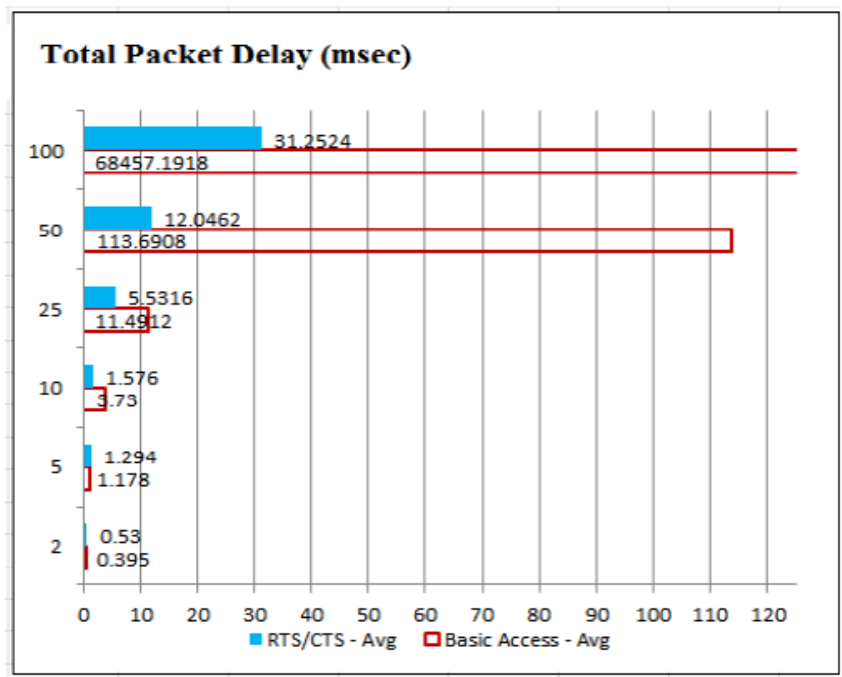

Fig. 4. Data Analysis: Total packet delay in milliseconds

\section{Delay Jitter}

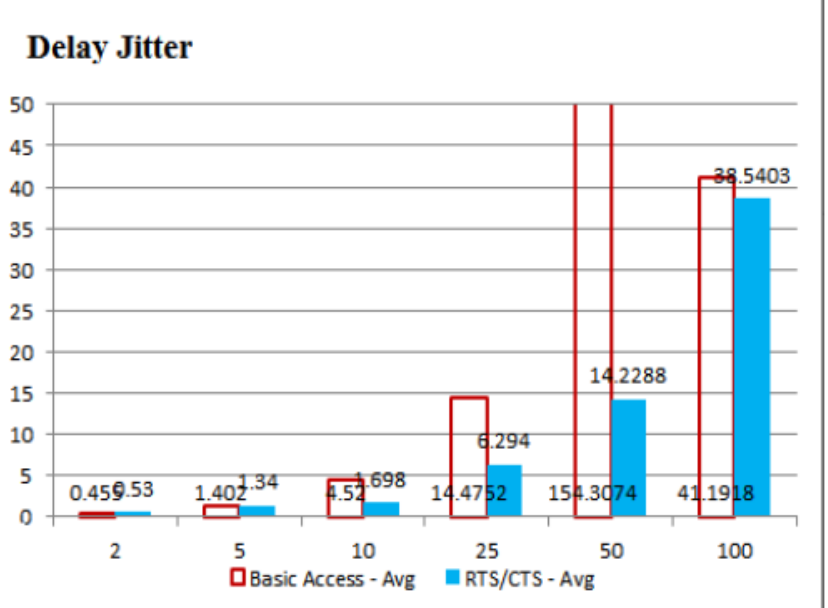

Fig. 5. Data Analysis: Jitter Delay

Fig 4. shows Total Packet Delay statistics, which is consistent with the Media Access Delay, as expected, since the total delay is a sum of the Media Access and other delays like Queueing Delay. The 2 node experiment showed that the delay using the RTS/CTS mechanism is marginally higher, but as the node concentration increased to 100 , the delay went far beyond the graph limits.

We can observe from fig. 5, a slight difference in the pattern for the delay jitter statistics. This experiment shows that the jitter for the 50 -node concentration experiment is way higher (and out of the graph bounds) than that reading for the 100-node experiment. This is an interesting anomaly that may need further analysis and research, but since the reading for Basic Access mode is still higher than the RTS/CTS mode, the observation is not considered to be of significance in the current study in progress.

\section{E. Retransmission Attempts}

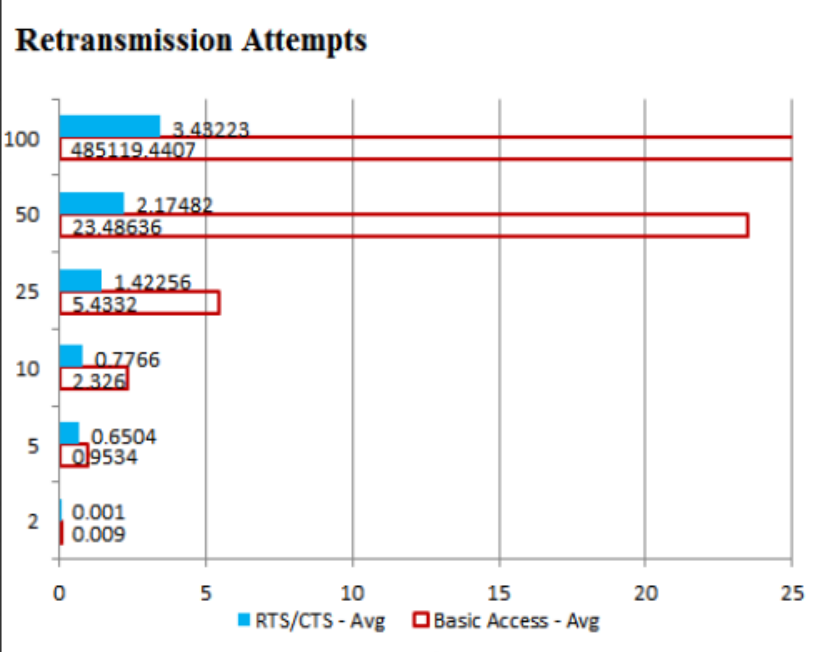

Fig. 6. Data Analysis: Retransmission Attempts 
We can see from fig. 6. that the Basic Access mode needs significantly higher retransmissions as expected, due to the expected higher collision rates. This chart clearly explains why all the other experiments showed significantly higher data for the experiments with more number of nodes. The extremely high number of retransmissions for the 100-node experiment adds to the delays of the 100-node experiments and thereby results in them shooting beyond the bounds of the graph.

TABLE I. DATA SUMMARY

\begin{tabular}{|c|c|c|c|c|c|}
\hline \multirow[b]{2}{*}{ Nodes } & \multicolumn{5}{|c|}{ Mean Data Analysis } \\
\hline & $\begin{array}{c}\begin{array}{c}\text { Run Time } \\
\text { (sec) }\end{array} \\
\end{array}$ & $\begin{array}{c}\text { Med Del } \\
(\mathrm{msec})\end{array}$ & $\begin{array}{c}\text { Total Delay } \\
\text { (msec) }\end{array}$ & Del Jitter & Retrans \\
\hline 2-BA & 4 & 0.395 & 0.395 & 0.455 & 0.009 \\
\hline 2-RC & 3 & 0.53 & 0.53 & 0.53 & 0.001 \\
\hline 5-BA & 10 & 1.178 & 1.178 & 1.402 & 0.9534 \\
\hline $5-\mathrm{RC}$ & 9 & 1.294 & 1.294 & 1.34 & 0.6504 \\
\hline 10-BA & 20 & 3.728 & 3.73 & 4.52 & 2.326 \\
\hline $10-\mathrm{RC}$ & 18 & 1.576 & 1.576 & 1.698 & 0.7766 \\
\hline 25-BA & 70 & 11.4284 & 11.4912 & 14.4752 & 5.4332 \\
\hline $25-\mathrm{RC}$ & 51 & 5.5292 & 5.5316 & 6.294 & 1.42256 \\
\hline 50-BA & 356 & 78.4292 & 113.6908 & 154.3074 & 23.48636 \\
\hline $50-\mathrm{RC}$ & 143 & 12.026 & 12.0462 & 14.2288 & 2.17482 \\
\hline $100-\mathrm{BA}$ & 2822 & 23847.3225 & 68457.1918 & 41.1918 & 485119.4407 \\
\hline $100-\mathrm{RC}$ & 577 & 30.9052 & 31.2524 & 38.5403 & 3.43223 \\
\hline & & c. Med D & $\begin{array}{l}\text { un Time: Time to ca } \\
\text { el: Media Access De }\end{array}$ & $\begin{array}{l}B A-\text { Basic } A c c e \\
\text { omplete simulati } \\
\text { elay, as a mean o }\end{array}$ & $\begin{array}{l}\text { ess, } R C-R T S / C T S \\
\text { ion run, in seconds } \\
\text { of the data samples }\end{array}$ \\
\hline
\end{tabular}

Table 1. shows the actual data plotted on the charts, showing the simulation run times and mean values for Media Access Delay, Total Delay, Delay Jitter and Retransmissions. As we can see, when the node concentration increases, the collision count is very high for the basic access mode in comparison to the CSMA/CA mode. This results in the increase of the total delay of transmission and thereby taking much longer to complete. The experiment sufficiently verifies that as the node concentration increases, the efficiency of using a protocol is more and more evident.

\section{CONCLUSION AND FUTURE WORK}

As we can see from the experiment results above, the introduction of a RTS/CTS based protocol yields good results for all cases we tested, from as low as 10 nodes to a 100 nodes in the same grid. Even though the smaller scenario shows us just a minor improvement of about $10 \%$, the largest node concentration experiment shows a huge improvement in performance of roughly 5 times. The total retransmission counts are suggestive and consistent with the rest of the experiment data. The retransmissions increase exponentially with node concentration, causing degradation to the network performance. This clearly indicates that the effect of collision is quite large when the concentration of nodes increases. It also gives us an indication of the effectiveness of the RTS/CTS in improving the overall performance of wireless $802.11 \mathrm{~b}$ and $802.11 \mathrm{~g}$ networks.

Hence, this experiment verifies the importance of the role of efficient protocols for high performance and effectiveness in network communication. Hence improvements in protocol design can have a good impact on the overall performance and therefore further research in improvement of protocols is highly beneficial for overall improvement of network communication. The impact of such improvements will be evidenced to a greater degree in small powered networking scenarios like wireless sensor networks and the like of it.

\section{REFERENCES}

[1] Mathew, Kuruvilla, and Biju Issac. "Ubiquitous Text Transfer Using Sound a Zero-Infrastructure Alternative for Simple Text Communication." Innovations and Advances in Computing, Informatics, Systems Sciences, Networking and Engineering. Springer International Publishing, 2015. 241-246.

[2] Ye, Wei, John Heidemann, and Deborah Estrin. "An energy-efficient MAC protocol for wireless sensor networks." INFOCOM 2002. Twenty-First Annual Joint Conference of the IEEE Computer and Communications Societies. Proceedings. IEEE. Vol. 3. IEEE, 2002.

[3] Wang, Guanghoug, et al. "Delay analysis of the IEEE 802.11 DCF." Personal, Indoor and Mobile Radio Communications, 2003. PIMRC 2003. 14th IEEE Proceedings on. Vol. 2. IEEE, 2003.

[4] Bruno, Raffaele, Marco Conti, and Enrico Gregori. "IEEE 802.11 optimal performances: RTS/CTS mechanism vs. basic access." Personal, Indoor and Mobile Radio Communications, 2002. The 13th IEEE International Symposium on. Vol. 4. IEEE, 2002.

[5] Bianchi, Giuseppe. "Performance analysis of the IEEE 802.11 distributed coordination function." Selected Areas in Communications, IEEE Journal on 18.3 (2000): 535-547.

[6] Nasipuri, Asis, Jun Zhuang, and Samir R. Das. "A multichannel CSMA MAC protocol for multihop wireless networks." Wireless Communications and Networking Conference, 1999. WCNC. 1999 IEEE. IEEE, 1999.

[7] Dean, Tamara. "Wireless Networking" in Network+ guide to networks. Cengage Learning, 2010.

[8] Xu, Kaixin, Mario Gerla, and Sang Bae. "Effectiveness of RTS/CTS handshake in IEEE 802.11 based ad hoc networks." Ad Hoc Networks 1.1 (2003): 107-123.

[9] Sobrinho, Joao Luis, Roland De Haan, and José Manuel Brázio "Why RTS-CTS is not your ideal wireless LAN multiple access protocol." Wireless Communications and Networking Conference, 2005 IEEE. Vol. 1. IEEE, 2005.

[10] Chatzimisios, P., A. C. Boucouvalas, and V. Vitsas. "Effectiveness of RTS/CTS handshake in IEEE 802.11 a wireless LANs." Electronics Letters 40.14 (2004): 915-916.

[11] Weinmiller, Jost, et al. "Analyzing the RTS/CTS mechanism in the DFWMAC media access protocol for wireless LANs." (1995)

[12] $\mathrm{Yu}, \mathrm{Chansu}$, et al. "Medium access control mechanisms in mobile ad hoc networks." Mobile Computing Handbook, CRC Press, Boca Raton, FL (2004).

[13] Ray, Saikat, Jeffrey B. Carruthers, and David Starobinski. "RTS/CTS-induced congestion in ad hoc wireless LANs." Wireless Communications and Networking, 2003. WCNC 2003. 2003 IEEE. Vol. 3. IEEE, 2003. 
[14] Tay, Y. C., and Kee Chaing Chua. "A capacity analysis for the IEEE 802.11 MAC protocol." Wireless networks 7.2 (2001): 159-171.

[15] Bianchi, Giuseppe. "IEEE 802.11-saturation throughput analysis." Communications Letters, IEEE 2.12 (1998): 318-320.

[16] Chatzimisios, Periklis, Anthony C. Boucouvalas, and Vasileios Vitsas. "Packet delay analysis of IEEE 802.11 MAC protocol." Electronics Letters 39.18 (2003): 1358-1359.
[17] Kuo, Wen-Kuang, Fang Liu, and C-C. Jay J. Kuo. "Enhanced backoff scheme in CSMA/CA for IEEE 802.11." AeroSense 2003. International Society for Optics and Photonics, 2003.

[18] Vassis, D., \& Zafeiris, V. (n.d.). Retrieved February 25, 2015, from http://www.pamvotis.org/ 